\title{
An Inverse Quadratic Eigenvalue Problem for Damped Structural Systems
}

\author{
Yongxin Yuan \\ Department of Mathematics, Jiangsu University of Science and Technology, Zhenjiang 212003, China
}

Correspondence should be addressed to Yongxin Yuan, yuanyx_703@163.com

Received 23 October 2007; Accepted 14 February 2008

Recommended by Angelo Luongo

\begin{abstract}
We first give the representation of the general solution of the following inverse quadratic eigenvalue problem (IQEP): given $\Lambda=\operatorname{diag}\left\{\lambda_{1}, \ldots, \lambda_{p}\right\} \in \mathbf{C}^{p \times p}, X=\left[x_{1}, \ldots, x_{p}\right] \in \mathbf{C}^{n \times p}$, and both $\Lambda$ and $X$ are closed under complex conjugation in the sense that $\lambda_{2 j}=\bar{\lambda}_{2 j-1} \in \mathbf{C}, x_{2 j}=\bar{x}_{2 j-1} \in \mathbf{C}^{n}$ for $j=1, \ldots, l$, and $\lambda_{k} \in \mathbf{R}, x_{k} \in \mathbf{R}^{n}$ for $k=2 l+1, \ldots, p$, find real-valued symmetric $(2 r+1)$-diagonal matrices $M, D$ and $K$ such that $M X \Lambda^{2}+D X \Lambda+K X=0$. We then consider an optimal approximation problem: given real-valued symmetric $(2 r+1)$-diagonal matrices $M_{a}, D_{a}, K_{a} \in \mathbf{R}^{n \times n}$, find $(\widehat{M}, \widehat{D}, \widehat{K}) \in \mathrm{S}_{\mathrm{E}}$ such that $\left\|\widehat{M}-M_{a}\right\|^{2}+\left\|\widehat{D}-D_{a}\right\|^{2}+\left\|\widehat{K}-K_{a}\right\|^{2}=\inf _{(M, D, K) \in \mathbf{S}_{\mathrm{E}}}\left(\left\|M-M_{a}\right\|^{2}+\left\|D-D_{a}\right\|^{2}+\left\|K-K_{a}\right\|^{2}\right)$, where $S_{\mathbf{E}}$ is the solution set of IQEP. We show that the optimal approximation solution $(\widehat{M}, \widehat{D}, \widehat{K})$ is unique and derive an explicit formula for it.
\end{abstract}

Copyright $(2008$ Yongxin Yuan. This is an open access article distributed under the Creative Commons Attribution License, which permits unrestricted use, distribution, and reproduction in any medium, provided the original work is properly cited.

\section{Introduction}

Throughout this paper, we will adopt the following notation. $\mathbf{C}^{m \times n}$ and $\mathbf{R}^{m \times n}$ denote the set of all $m \times n$ complex and real matrices, respectively. $\mathbf{S R}^{n \times n}$ denotes the set of all symmetric matrices in $\mathbf{R}^{n \times n}$. $A^{T}$ and $A^{+}$stand for the transpose and the Moore-Penrose generalized inverse of a real matrix $A$. $I_{n}$ represents the identity matrix of size $n ; \bar{\alpha}$ denotes the conjugate of the complex number $\alpha$. For $A, B \in \mathbf{R}^{m \times n}$, an inner product in $\mathbf{R}^{m \times n}$ is defined by $(A, B)=\operatorname{trace}\left(B^{T} A\right)$, then $\mathbf{R}^{m \times n}$ is a Hilbert space. The matrix norm $\|\cdot\|$ induced by the inner product is the Frobenius norm. Given two matrices $A=\left[a_{i j}\right] \in \mathbf{R}^{m \times n}$ and $B=\left[b_{i j}\right] \in \mathbf{R}^{p \times q}$, the Kronecker product of $A$ and $B$ is defined by $A \otimes B=\left[a_{i j} B\right] \in \mathbf{R}^{m p \times n q}$, and the stretching function $\operatorname{Vec}(A)$ is defined by $\operatorname{Vec}(A)=\left[a_{1}^{T}, a_{2}^{T}, \ldots, a_{n}^{T}\right]^{T} \in \mathbf{R}^{m n}$, where $a_{i}, i=1, \ldots, n$, is the $i$ th column vector of $A$. Furthermore, for a matrix $A \in \mathbf{R}^{m \times n}$, let $E_{A}$ and $F_{A}$ stand for the two orthogonal projectors $E_{A}=I_{m}-A A^{+}$and $F_{A}=I_{n}-A^{+} A$. 
Using finite element techniques, vibrating structures such as beams, buildings, bridges, highways, and large space structures can be discretized to matrix second-order models (referred to as analytical models). A matrix second-order model of the free motion of a vibrating system is a system of differential equations of the form

$$
M_{a} \ddot{x}(t)+D_{a} \dot{x}(t)+K_{a} x(t)=0,
$$

where $M_{a}, D_{a}$, and $K_{a}$ are the $n \times n$ analytical mass, damping, and stiffness matrices. The system represented by (1.1) is called damped structural system. It is well known that all solutions of (1.1) can be obtained via the algebraic equation

$$
\left(\lambda^{2} M_{a}+\lambda D_{a}+K_{a}\right) x=0
$$

Complex numbers $\lambda$ and nonzero complex vectors $x$ for which this relation holds are, respectively, the eigenvalues and eigenvectors of the system. It is known that (1.2) has $2 n$ finite eigenvalues over the complex field, provided that the leading matrix coefficient $M_{a}$ is nonsingular.

Due to the complexity of the structure, the finite element model is only an approximation to the practical structure. On the other hand, a part of the natural frequencies (eigenvalues) and corresponding mode shapes (eigenvectors) of the structure can be obtained experimentally by performing vibration tests [1]. Generally speaking, very often natural frequencies and mode shapes of an analytical model described by (1.2) do not match very well with experimentally measured frequencies and mode shapes. Thus, engineers would like to improve the analytical model of the structure such that the updated model predicts the observed dynamic behavior. Then, the updated model may be considered to be a better dynamic representation of the structure. This model can be used with greater confidence for the analysis of the structure under different boundary conditions or with physical structural changes.

For undamped systems (i.e., $D_{a}=0$ ), various techniques for updating mass and stiffness matrices using measured response data have been discussed by Baruch [2], Baruch and BarItzhack [3], Berman [4], Berman and Nagy [5], and Wei [6, 7]. For damped structural systems, the theory and computation were first proposed by Friswell et al. [8, 9]; they applied the ideas in $[2,3]$ to minimize changes between the analytical and updated model subject to the spectral constraints. Kuo et al. [10] have recently proposed a direct method to close the weaknesses in [8] which seems more efficient and reliable. All these existing methods can reproduce the given set of measured data while updated matrices symmetry, but the connectivity of the original finite element model is not necessarily preserved, causing the addition of unwanted load paths.

The purpose of the work presented in this paper is to develop a new method for finite element model updating problems which preserves the connectivity of the original model. Assume that $M_{a}, D_{a}$, and $K_{a}$ are real-valued symmetric $(2 r+1)$-diagonal matrices. Thus, the problem of updating mass, damping, and stiffness matrices simultaneously can be mathematically formulated as follows.

Problem IQEP. Given matrices $\Lambda=\operatorname{diag}\left\{\lambda_{1}, \ldots, \lambda_{p}\right\} \in \mathbf{C}^{p \times p}, X=\left[x_{1}, \ldots, x_{p}\right] \in \mathbf{C}^{n \times p}$, and both $\Lambda$ and $X$ are closed under complex conjugation in the sense that $\lambda_{2 j}=\bar{\lambda}_{2 j-1} \in \mathbf{C}, x_{2 j}=\bar{x}_{2 j-1} \in \mathbf{C}^{n}$ for $j=1, \ldots, l$, and $\lambda_{k} \in \mathbf{R}, x_{k} \in \mathbf{R}^{n}$ for $k=2 l+1, \ldots, p$, find real-valued symmetric $(2 r+1)$ diagonal matrices $M, D$, and $K$ such that

$$
M X \Lambda^{2}+D X \Lambda+K X=0 .
$$


Problem II. Let $\mathrm{S}_{\mathrm{E}}$ be the solution set of IQEP. Find $(\widehat{M}, \widehat{D}, \widehat{K}) \in \mathrm{S}_{\mathrm{E}}$ such that

$$
\left\|\widehat{M}-M_{a}\right\|^{2}+\left\|\widehat{D}-D_{a}\right\|^{2}+\left\|\widehat{K}-K_{a}\right\|^{2}=\inf _{(M, D, K) \in S_{\mathrm{E}}}\left(\left\|M-M_{a}\right\|^{2}+\left\|D-D_{a}\right\|^{2}+\left\|K-K_{a}\right\|^{2}\right) .
$$

The paper is organized as follows. In Section 2, using the Kronecker product and stretching function of matrices, we give an explicit representation of the solution set $\mathbf{S}_{\mathrm{E}}$ of Problem IQEP. In Section 3, we show that there exists a unique solution in Problem II and present the expression of the unique solution $(\widehat{M}, \widehat{D}, \widehat{K})$. Finally, in Section 4 , a numerical algorithm to acquire the optimal approximation solution under the Frobenius norm sense is described, and a numerical example is provided.

\section{The solution of Problem IQEP}

To begin with, we introduce a lemma [11].

Lemma 2.1. If $L \in \mathbf{R}^{m \times q}, b \in \mathbf{R}^{m}$, then $L y=b$ has a solution $y \in \mathbf{R}^{q}$ if and only if $L L^{+} b=b$. In this case, the general solution of the equation can be described as $y=L^{+} b+\left(I_{q}-L^{+} L\right) z$, where $z \in \mathbf{R}^{q}$ is an arbitrary vector.

Let $S_{0}$ be the set of all $n \times n$ real-valued symmetric $(2 r+1)$-diagonal matrices, then $S_{0}$ is a linear subspace of $\mathbf{S R}^{n \times n}$, and the dimension of $S_{0}$ is $N=(1 / 2)(2 n-r)(r+1)$.

Define $Y_{i j}$ as

$$
Y_{i j}= \begin{cases}\frac{\sqrt{2}}{2}\left(e_{i} e_{j}^{T}+e_{j} e_{i}^{T}\right), & i=1, \ldots, n-1 ; j=i+1, \ldots, t_{i}, \\ e_{i} e_{i}^{T}, & i=j=1, \ldots, n,\end{cases}
$$

where $t_{i}=\min \{i+r, n\}$ and $e_{i}, i=1, \ldots, n$, is the $i$ th column vector of the identity matrix $I_{n}$. It is easy to verify that $\left\{Y_{i j}\right\}$ forms an orthonormal basis of the subspace $S_{0}$, that is,

$$
\left(Y_{i j}, Y_{k l}\right)= \begin{cases}0, & i \neq k \text { or } j \neq l \\ 1, & i=k \text { and } j=l\end{cases}
$$

Now, if $M, D, K \in \mathbf{S R}^{n \times n}$ are $(2 r+1)$-diagonal matrices, then $M, D, K$ can be expressed as

$$
M=\sum_{i, j} \alpha_{i j} Y_{i j}, \quad D=\sum_{i, j} \beta_{i j} Y_{i j}, \quad K=\sum_{i, j} Y_{i j} Y_{i j}
$$

where the real numbers $\alpha_{i j}, \beta_{i j}, \gamma_{i j}, i=1, \ldots, n ; j=i, \ldots, t_{i}, t_{i}=\min \{i+r, n\}$, are yet to be determined.

Define a matrix $T_{p}$ as

$$
T_{p}=\operatorname{diag}\left\{\frac{1}{\sqrt{2}}\left[\begin{array}{cc}
1 & -i \\
1 & i
\end{array}\right], \ldots, \frac{1}{\sqrt{2}}\left[\begin{array}{cc}
1 & -i \\
1 & i
\end{array}\right], I_{p-2 l}\right\} \in \mathbf{C}^{p \times p},
$$


where $i=\sqrt{-1}$. It is easy to verify that $T_{p}$ is a unitary matrix, that is, $\bar{T}_{p}^{T} T_{p}=I_{p}$. Using this transformation matrix, we have

$$
\begin{aligned}
& \tilde{\Lambda}=\bar{T}_{p}^{T} \Lambda T_{p}=\operatorname{diag}\left\{\left[\begin{array}{cc}
\zeta_{1} & \eta_{1} \\
-\eta_{1} & \zeta_{1}
\end{array}\right], \ldots,\left[\begin{array}{cc}
\zeta_{2 l-1} & \eta_{2 l-1} \\
-\eta_{2 l-1} & \zeta_{2 l-1}
\end{array}\right], \lambda_{2 l+1}, \ldots, \lambda_{p}\right\} \in \mathbf{R}^{p \times p}, \\
& \tilde{X}=X T_{p}=\left[\sqrt{2} y_{1}, \sqrt{2} z_{1}, \ldots, \sqrt{2} y_{2 l-1}, \sqrt{2} z_{2 l-1}, x_{2 l+1}, \ldots, x_{p}\right] \in \mathbf{R}^{n \times p},
\end{aligned}
$$

where $\zeta_{j}$ and $\eta_{j}$ are, respectively, the real part and the imaginary part of the complex number $\lambda_{j} ; y_{j}$ and $z_{j}$, are, respectively, the real part and the imaginary part of the complex vector $x_{j}$ for $j=1,3, \ldots, 2 l-1$.

It follows from (2.5) and (2.6) that (1.3) can be equivalently written as

$$
M \tilde{X} \tilde{\Lambda}^{2}+D \tilde{X} \tilde{\Lambda}+K \tilde{X}=0
$$

Substituting (2.3) into (2.7), we have

$$
\sum_{i, j} \alpha_{i j} Y_{i j} \tilde{X} \tilde{\Lambda}^{2}+\sum_{i, j} \beta_{i j} Y_{i j} \tilde{X} \tilde{\Lambda}+\sum_{i, j} \gamma_{i j} Y_{i j} \tilde{X}=0
$$

When setting

$$
\begin{aligned}
\alpha= & {\left[\alpha_{11}, \ldots, \alpha_{1, r+1}, \ldots, \alpha_{n-r, n-r}, \ldots, \alpha_{n-r, n}, \ldots, \alpha_{n-1, n-1}, \alpha_{n-1, n}, \alpha_{n, n}\right]^{T}, } \\
\beta= & {\left[\beta_{11}, \ldots, \beta_{1, r+1}, \ldots, \beta_{n-r, n-r}, \ldots, \beta_{n-r, n}, \ldots, \beta_{n-1, n-1}, \beta_{n-1, n}, \beta_{n, n}\right]^{T}, } \\
\gamma= & {\left[\gamma_{11}, \ldots, \gamma_{1, r+1}, \ldots, \gamma_{n-r, n-r}, \ldots, \gamma_{n-r, n}, \ldots, \gamma_{n-1, n-1}, \gamma_{n-1, n}, \gamma_{n, n}\right]^{T}, } \\
G= & {\left[\operatorname{Vec}\left(Y_{11}\right), \ldots, \operatorname{Vec}\left(Y_{1, r+1}\right), \ldots, \operatorname{Vec}\left(Y_{n-r, n-r}\right), \ldots,\right.} \\
& \left.\operatorname{Vec}\left(Y_{n-r, n}\right), \ldots, \operatorname{Vec}\left(Y_{n-1, n-1}\right), \operatorname{Vec}\left(Y_{n-1, n}\right), \operatorname{Vec}\left(Y_{n, n}\right)\right] \in \mathbf{R}^{n^{2} \times N}, \\
A= & \left(\left(\tilde{\Lambda}^{2}\right)^{T} \tilde{X}^{T} \otimes I_{n}\right) G, \quad B=\left(\tilde{\Lambda}^{T} \tilde{X}^{T} \otimes I_{n}\right) G, \quad C=\left(\tilde{X}^{T} \otimes I_{n}\right) G .
\end{aligned}
$$

We see that (2.8) is equivalent to

$$
A \alpha+B \beta+C \gamma=0
$$

It follows from Lemma 2.1 that (2.10) with unknown vector $\alpha$ has a solution if and only if

$$
E_{A} B \beta=-E_{A} C \gamma .
$$

Using Lemma 2.1 again, we know that (2.11) with respect to $\beta$ has a solution if and only if

$$
E_{H} E_{A} C r=0,
$$

where $H=E_{A} B$. It follows from Lemma 2.1 that (2.12) with respect to $\gamma$ is always solvable, and the general solution to the equation is

$$
\gamma=F_{W} u
$$


where $W=E_{H} E_{A} C$, and $u \in \mathbf{R}^{N}$ is an arbitrary vector. Substituting (2.13) into (2.11) and applying Lemma 2.1, we obtain

$$
\beta=-H^{+} E_{A} C F_{W} u+F_{H} v
$$

where $v \in \mathbf{R}^{N}$ is an arbitrary vector. Inserting (2.14) and (2.13) into (2.10) yields

$$
\alpha=A^{+} B H^{+} E_{A} C F_{W} u-A^{+} B F_{H} v-A^{+} C F_{W} u+F_{A} q,
$$

where $q \in \mathbf{R}^{N}$ is an arbitrary vector.

As a summary of the above discussion, we have proved the following result.

Theorem 2.2. Suppose that $\Lambda=\operatorname{diag}\left\{\lambda_{1}, \ldots, \lambda_{p}\right\} \in \mathbf{C}^{p \times p}, X=\left[x_{1}, \ldots, x_{p}\right] \in \mathbf{C}^{n \times p}$, and both $\Lambda$ and $X$ are closed under complex conjugation. The real matrices $\tilde{\Lambda}$ and $\tilde{X}$ are given by (2.5) and (2.6). Let $\left\{Y_{i j}\right\}, G, A, B, C$ be given as in (2.1), (2.9). Write $N=(1 / 2)(2 n-r)(r+1), H=E_{A} B, W=E_{H} E_{A} C$. Then the solution set $\mathbf{S}_{\mathrm{E}}$ of problem IQEP can be expressed as

$$
\mathbf{S}_{\mathrm{E}}=\left\{(M, D, K) \in \mathbf{S R}^{n \times n} \times \mathbf{S R}^{n \times n} \times \mathbf{S R}^{n \times n} \mid M=S\left(\alpha \otimes I_{n}\right), D=S\left(\beta \otimes I_{n}\right), K=S\left(\gamma \otimes I_{n}\right)\right\},
$$

where

$$
S=\left[Y_{11}, \ldots, Y_{1, r+1}, \ldots, Y_{n-r, n-r}, \ldots, Y_{n-r, n}, \ldots, Y_{n-1, n-1}, Y_{n-1, n}, Y_{n, n}\right] \in \mathbf{R}^{n \times n N}
$$

$\alpha, \beta, \gamma$ are, respectively, given by (2.15), (2.14), and (2.13) with $u, v, q \in \mathbf{R}^{N}$ being arbitrary vectors.

\section{The solution of Problem II}

It follows from Theorem 2.2 that the set $S_{\mathrm{E}}$ is always nonempty. It is easy to verify that $\mathrm{S}_{\mathrm{E}}$ is a closed convex subset of $\mathbf{S R}^{n \times n} \times \mathbf{S R}^{n \times n} \times \mathbf{S R}^{n \times n}$. From the best approximation theorem (see [12]), we know there exists a unique solution $(\widehat{M}, \widehat{D}, \widehat{K})$ in $S_{\mathrm{E}}$ such that (1.4) holds.

We now focus our attention on seeking the unique solution $(\widehat{M}, \widehat{D}, \widehat{K})$ in $\mathrm{S}_{\mathrm{E}}$. For the realvalued symmetric $(2 r+1)$-diagonal matrices $M_{a}, D_{a}$, and $K_{a}$, it is easily seen that $M_{a}, D_{a}$, and $K_{a}$ can be expressed as the linear combinations of the orthonormal basis $\left\{Y_{i j}\right\}$, that is,

$$
M_{a}=\sum_{i, j} \delta_{i j} Y_{i j}, \quad D_{a}=\sum_{i, j} \xi_{i j} Y_{i j}, \quad K_{a}=\sum_{i, j} \varphi_{i j} Y_{i j},
$$

where $\delta_{i j}, \xi_{i j}, \varphi_{i j}, \quad i=1, \ldots, n ; j=i, \ldots, t_{i}, t_{i}=\min \{i+r, n\}$, are uniquely determined by the elements of $M_{a}, D_{a}$, and $K_{a}$. Let

$$
\begin{aligned}
\delta & =\left[\delta_{11}, \ldots, \delta_{1, r+1}, \ldots, \delta_{n-r, n-r}, \ldots, \delta_{n-r, n}, \ldots, \delta_{n-1, n-1}, \delta_{n-1, n}, \delta_{n, n}\right]^{T}, \\
\xi & =\left[\xi_{11}, \ldots, \xi_{1, r+1}, \ldots, \xi_{n-r, n-r}, \ldots, \xi_{n-r, n}, \ldots, \xi_{n-1, n-1}, \xi_{n-1, n}, \xi_{n, n}\right]^{T}, \\
\varphi & =\left[\varphi_{11}, \ldots, \varphi_{1, r+1}, \ldots, \varphi_{n-r, n-r}, \ldots, \varphi_{n-r, n}, \ldots, \varphi_{n-1, n-1}, \varphi_{n-1, n}, \varphi_{n, n}\right]^{T} .
\end{aligned}
$$


Then, for any triple of matrices $(M, D, K) \in \mathbf{S}_{\mathrm{E}}$ in (2.16), by the relations of (2.2) and (3.1), we see that

$$
\begin{aligned}
f= & \left\|M-M_{a}\right\|^{2}+\left\|D-D_{a}\right\|^{2}+\left\|K-K_{a}\right\|^{2} \\
= & \left\|\sum_{i, j}\left(\alpha_{i j}-\delta_{i j}\right) Y_{i j}\right\|^{2}+\left\|\sum_{i, j}\left(\beta_{i j}-\xi_{i j}\right) Y_{i j}\right\|^{2}+\left\|\sum_{i, j}\left(\gamma_{i j}-\varphi_{i j}\right) Y_{i j}\right\|^{2} \\
= & \left(\sum_{i, j}\left(\alpha_{i j}-\delta_{i j}\right) Y_{i j}, \sum_{i, j}\left(\alpha_{i j}-\delta_{i j}\right) Y_{i j}\right)+\left(\sum_{i, j}\left(\beta_{i j}-\xi_{i j}\right) Y_{i j}, \sum_{i, j}\left(\beta_{i j}-\xi_{i j}\right) Y_{i j}\right) \\
& +\left(\sum_{i, j}\left(\gamma_{i j}-\varphi_{i j}\right) Y_{i j}, \sum_{i, j}\left(\gamma_{i j}-\varphi_{i j}\right) Y_{i j}\right) \\
= & \sum_{i, j}\left(\alpha_{i j}-\delta_{i j}\right)\left(Y_{i j}, \sum_{i, j}\left(\alpha_{i j}-\delta_{i j}\right) Y_{i j}\right)+\sum_{i, j}\left(\beta_{i j}-\xi_{i j}\right)\left(Y_{i j}, \sum_{i, j}\left(\beta_{i j}-\xi_{i j}\right) Y_{i j}\right) \\
& +\sum_{i, j}\left(\gamma_{i j}-\varphi_{i j}\right)\left(Y_{i j}, \sum_{i, j}\left(\gamma_{i j}-\varphi_{i j}\right) Y_{i j}\right) \\
= & \sum_{i, j}\left(\alpha_{i j}-\delta_{i j}\right)^{2}+\sum_{i, j}\left(\beta_{i j}-\xi_{i j}\right)^{2}+\sum_{i, j}\left(\gamma_{i j}-\varphi_{i j}\right)^{2} \\
= & \|\alpha-\delta\|^{2}+\|\beta-\xi\|^{2}+\|\gamma-\varphi\|^{2} .
\end{aligned}
$$

Substituting (2.13), (2.14), and (2.15) into the relation of $f$, we have

$$
\begin{aligned}
f= & \left\|J u-A^{+} B F_{H} v+F_{A} q-\delta\right\|^{2}+\left\|-L u+F_{H} v-\xi\right\|^{2}+\left\|F_{W} u-\varphi\right\|^{2} \\
= & u^{T} J^{T} J u-2 u^{T} J^{T} A^{+} B F_{H} v-2 u^{T} J^{T} \delta+v^{T} F_{H} B^{T}\left(A^{+}\right)^{T} A^{+} B F_{H} v+2 v^{T} F_{H} B^{T}\left(A^{+}\right)^{T} \delta \\
& +q^{T} F_{A} q-2 q^{T} F_{A} \delta+\delta^{T} \delta+u^{T} L^{T} L u+2 u^{T} L^{T} \xi+v^{T} F_{H} v-2 v^{T} F_{H} \xi+\xi^{T} \xi \\
& +u^{T} F_{W} u-2 u^{T} F_{W} \varphi+\varphi^{T} \varphi,
\end{aligned}
$$

where $L=H^{+} E_{A} C F_{W}, J=A^{+} B L-A^{+} C F_{W}$. Therefore,

$$
\begin{aligned}
& \frac{\partial f}{\partial u}=2 J^{T} J u-2 J^{T} A^{+} B F_{H} v-2 J^{T} \delta+2 L^{T} L u+2 L^{T} \xi+2 F_{W} u-2 F_{W} \varphi \\
& \frac{\partial f}{\partial v}=-2 F_{H} B^{T}\left(A^{+}\right)^{T} J u+2 F_{H} B^{T}\left(A^{+}\right)^{T} A^{+} B F_{H} v+2 F_{H} B^{T}\left(A^{+}\right)^{T} \delta+2 F_{H} v-2 F_{H} \xi \\
& \frac{\partial f}{\partial q}=2 F_{A} q-2 F_{A} \delta
\end{aligned}
$$


Clearly, $f=\left\|M-M_{a}\right\|^{2}+\left\|D-D_{a}\right\|^{2}+\left\|K-K_{a}\right\|^{2}=\min$ if and only if

$$
\frac{\partial f}{\partial u}=0, \quad \frac{\partial f}{\partial v}=0, \quad \frac{\partial f}{\partial q}=0 .
$$

Note that $L F_{W}=L, J F_{W}=J$. Therefore, $\partial f / \partial u=0$ implies that

$$
F_{W} u=P^{-1} J^{T} A^{+} B F_{H} v+P^{-1}\left(J^{T} \delta-L^{T} \xi+F_{W} \varphi\right),
$$

where $P=J^{T} J+L^{T} L+I_{N}$. Substituting (3.7) into $\partial f / \partial v=0$ yields

$$
F_{H} v=Q^{+} F_{H} B^{T}\left(A^{+}\right)^{T} J P^{-1}\left(J^{T} \delta-L^{T} \xi+F_{W} \varphi\right)-Q^{+}\left(F_{H} B^{T}\left(A^{+}\right)^{T} \delta-F_{H} \xi\right),
$$

where $Q=I_{N}+F_{H} B^{T}\left(A^{+}\right)^{T} A^{+} B F_{H}-F_{H} B^{T}\left(A^{+}\right)^{T} J P^{-1} J^{T} A^{+} B F_{H}$.

Clearly, $\partial f / \partial q=0$ is equivalent to

$$
F_{A} q=F_{A} \delta
$$

Upon substituting (3.7) and (3.9) into (2.13), (2.14), and (2.15), we obtain

$$
\begin{aligned}
& \widehat{\alpha}=\left(J P^{-1} J^{T} A^{+} B-A^{+} B\right) F_{H} v+J P^{-1}\left(J^{T} \delta-L^{T} \xi+F_{W} \varphi\right)+F_{A} \delta, \\
& \widehat{\beta}=\left(I_{N}-L P^{-1} J^{T} A^{+} B\right) F_{H} v-L P^{-1}\left(J^{T} \delta-L^{T} \xi+F_{W} \varphi\right), \\
& \widehat{\gamma}=P^{-1} J^{T} A^{+} B F_{H} v+P^{-1}\left(J^{T} \delta-L^{T} \xi+F_{W} \varphi\right),
\end{aligned}
$$

where $F_{H} v$ is given by (3.8).

By now, we have proved the following result.

Theorem 3.1. Let the real-valued symmetric $(2 r+1)$-diagonal matrices $M_{a}, D_{a}$, and $K_{a}$ be given. Then, Problem II has a unique solution, and the unique solution of Problem II can be expressed as

$$
\widehat{M}=S\left(\widehat{\alpha} \otimes I_{n}\right), \widehat{D}=S\left(\widehat{\beta} \otimes I_{n}\right), \widehat{K}=S\left(\widehat{\gamma} \otimes I_{n}\right),
$$

where $\widehat{\alpha}, \widehat{\beta}$, and $\widehat{\gamma}$ are given by (3.10).

\section{A numerical example}

Based on Theorems 2.2 and 3.1, we can describe an algorithm for solving problem IQEP and Problem II as follows.

Example 4.1. Consider a five-DOF system modelled analytically with mass, damping, and stiffness matrices given by

$$
M_{a}=\left[\begin{array}{lllll}
2 & 1 & 0 & 0 & 0 \\
1 & 4 & 1 & 0 & 0 \\
0 & 1 & 4 & 1 & 0 \\
0 & 0 & 1 & 4 & 1 \\
0 & 0 & 0 & 1 & 4
\end{array}\right], \quad D_{a}=\left[\begin{array}{ccccc}
11.0 & -8.0 & 0 & 0 & 0 \\
-8.0 & 14.0 & -3.5 & 0 & 0 \\
0 & -3.5 & 13.0 & -7.8 & 0 \\
0 & 0 & -7.8 & 13.5 & -9.0 \\
0 & 0 & 0 & -9.0 & 15.4
\end{array}\right], \quad K_{a}=\left[\begin{array}{ccccc}
100 & -20 & 0 & 0 & 0 \\
-20 & 120 & -35 & 0 & 0 \\
0 & -35 & 80 & -12 & 0 \\
0 & 0 & -12 & 95 & -40 \\
0 & 0 & 0 & -40 & 124
\end{array}\right] .
$$




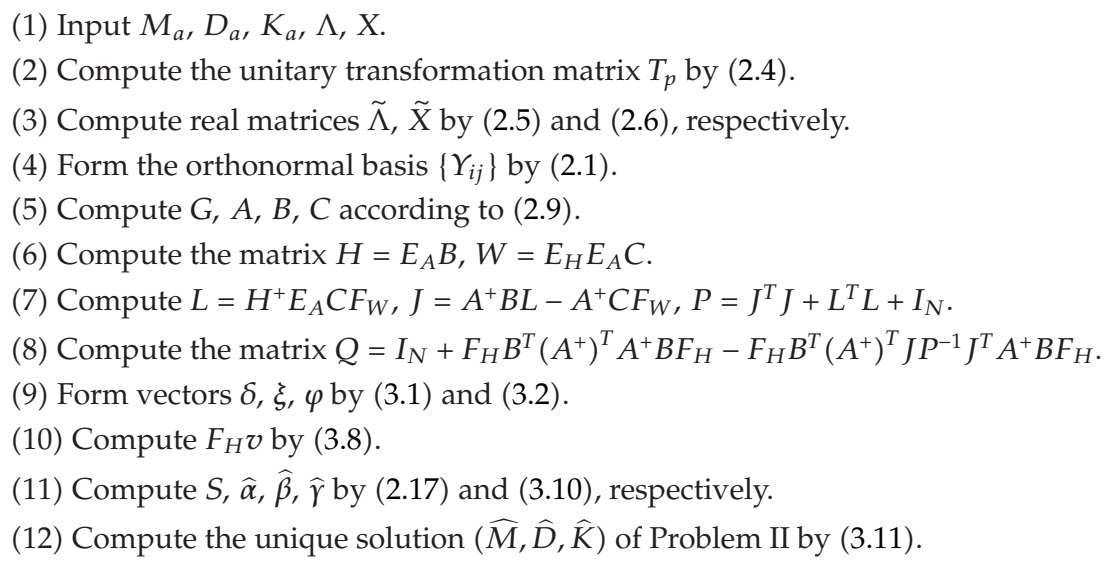

\section{Algorithm 1}

That is, $M_{a}, D_{a}, K_{a}$ are symmetric 3-diagonal matrices. The measured eigenvalue and eigenvector matrices $\Lambda=\operatorname{diag}\left\{\lambda_{1}, \lambda_{2}, \lambda_{3}, \lambda_{4}\right\}$ and $X=\left[x_{1}, x_{2}, x_{3}, x_{4}\right]$ are given by

$$
\begin{aligned}
\Lambda & =\operatorname{diag}\{-3.9525+6.8578 i,-3.9525-6.8578 i,-0.1622+3.3465 i,-0.1622-3.3465 i\}, \\
X & =\left[\begin{array}{cccc}
0.1099+0.4083 i & 0.1099-0.4083 i & -0.0283+0.1570 i & -0.0283-0.1570 i \\
0.0684-0.1754 i & 0.0684+0.1754 i & 0.0525+0.3742 i & 0.0525-0.3742 i \\
-0.1272-0.2703 i & -0.1272+0.2703 i & 0.0594+0.6146 i & 0.0594-0.6146 i \\
0.3403+0.5185 i & 0.3403-0.5185 i & -0.1132+0.5663 i & -0.1132-0.5663 i \\
-0.3783-0.4108 i & -0.3783+0.4108 i & -0.0862+0.3309 i & -0.0862-0.3309 i
\end{array}\right] .
\end{aligned}
$$

Using Algorithm 1, we obtain the unique solution of Problem II as follows:

$$
\begin{aligned}
\widehat{M} & =\left[\begin{array}{ccccc}
1.6010 & 0.3152 & 0 & 0 & 0 \\
0.3152 & 2.7909 & 1.5318 & 0 & 0 \\
0 & 1.5318 & 2.9332 & 0.9771 & 0 \\
0 & 0 & 0.9771 & 3.7779 & 1.2094 \\
0 & 0 & 0 & 1.2094 & 3.6053
\end{array}\right], \\
\widehat{D} & =\left[\begin{array}{cccccc}
9.0659 & -6.4506 & 0 & 0 & 0 & \\
-6.4506 & -5.5001 & 7.3677 & 0 & 0 \\
0 & 7.3677 & 4.1831 & -6.4524 & 0 \\
0 & 0 & -6.4524 & 10.2736 & -5.8430 \\
0 & 0 & 0 & -5.8430 & 10.3568
\end{array}\right], \\
\widehat{K} & =\left[\begin{array}{cccccc}
98.7229 & -25.5781 & 0 & 0 & 0 \\
-25.5781 & 124.0044 & -32.9787 & 0 & 0 \\
0 & -32.9787 & 77.7282 & -12.0909 & 0 \\
0 & 0 & -12.0909 & 99.5825 & -32.9904 \\
0 & 0 & 0 & -32.9904 & 120.7917
\end{array}\right] .
\end{aligned}
$$


We define the residual as

$$
\operatorname{res}\left(\lambda_{i}, x_{i}\right)=\left\|\left(\lambda_{i}^{2} \widehat{M}+\lambda_{i} \widehat{D}+\widehat{K}\right) x_{i}\right\|
$$

and the numerical results shown in the following table.

\begin{tabular}{lcccc}
\hline$\left(\lambda_{i}, x_{i}\right)$ & $\left(\lambda_{1}, x_{1}\right)$ & $\left(\lambda_{2}, x_{2}\right)$ & $\left(\lambda_{3}, x_{3}\right)$ & $\left(\lambda_{4}, x_{4}\right)$ \\
\hline $\operatorname{res}\left(\lambda_{i}, x_{i}\right)$ & $1.9584 \mathrm{e}-013$ & $1.9584 \mathrm{e}-013$ & $9.0344 \mathrm{e}-014$ & $9.0344 \mathrm{e}-014$ \\
\hline
\end{tabular}

Therefore, the prescribed eigenvalues (the diagonal elements of the matrix $\Lambda$ ) and eigenvectors (the column vectors of the matrix $X)$ are embedded in the new model $\left(\lambda^{2} \widehat{M}+\lambda \widehat{D}+\widehat{K}\right) x=$ 0 and the updated matrices $\widehat{M}, \widehat{D}, \widehat{K}$ are also symmetric 3-diagonal matrices, which implies that the structural connectivity information of the analytical model is preserved.

\section{References}

[1] D. H. F. Chu, Modal Testing and Modal Refinement, American Society of Mechanical Engineers, New York, NY, USA, 1983.

[2] M. Baruch, "Optimization procedure to correct stiffness and flexibility matrices using vibration tests," AIAA Journal, vol. 16, no. 11, pp. 1208-1210, 1978.

[3] M. Baruch and I. Y. Bar-Itzhack, "Optimal weighted orthogonalization of measured modes," AIAA Journal, vol. 16, no. 4, pp. 346-351, 1978.

[4] A. Berman, "Mass matrix correction using an incomplete set of measured modes," AIAA Journal, vol. 17, no. 10, pp. 1147-1148, 1979.

[5] A. Berman and E. J. Nagy, "Improvement of a large analytical model using test data," AIAA Journal, vol. 21, no. 8, pp. 1168-1173, 1983.

[6] F.-S. Wei, "Stiffness matrix correction from incomplete test data," AIAA Journal, vol. 18, no. 10, pp. 1274-1275, 1980.

[7] F.-S. Wei, "Mass and stiffness interaction effects in analytical model modification," AIAA Journal, vol. 28, no. 9, pp. 1686-1688, 1990.

[8] M. I. Friswell, D. J. Inman, and D. F. Pilkey, "The direct updating of damping and stiffness matrices," AIAA Journal, vol. 36, no. 3, pp. 491-493, 1998.

[9] D. F. Pilkey, "Computation of a damping matrix for finite element model updating," Ph.D. thesis, Department of Engineering Mechanics, Virginia Polytechnic Institute and State University, Blacksburg, Va, USA, 1998.

[10] Y.-C. Kuo, W.-W. Lin, and S.-F. Xu, "New methods for finite element model updating problems," AIAA Journal, vol. 44, no. 6, pp. 1310-1316, 2006.

[11] A. Ben-Israel and T. N. E. Greville, Generalized Inverses: Theory and Applications, Pure and Applied Mathematic, John Wiley \& Sons, New York, NY, USA, 1974.

[12] J.-P. Aubin, Applied Functional Analysis, John Wiley \& Sons, New York, NY, USA, 1979. 


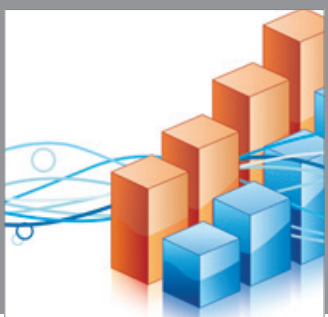

Advances in

Operations Research

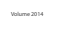

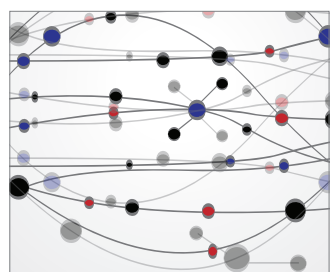

\section{The Scientific} World Journal
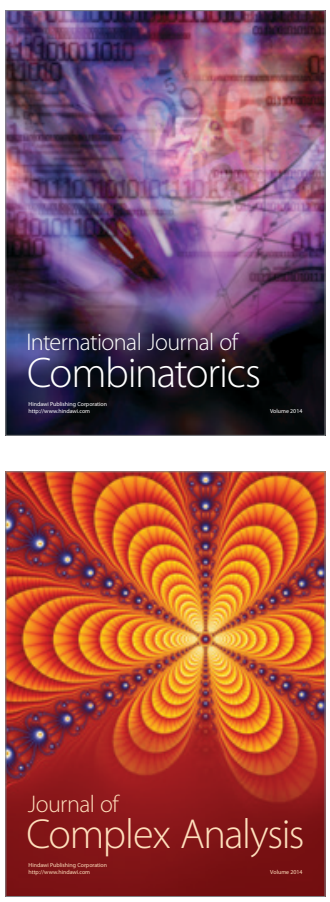

International Journal of

Mathematics and

Mathematical

Sciences
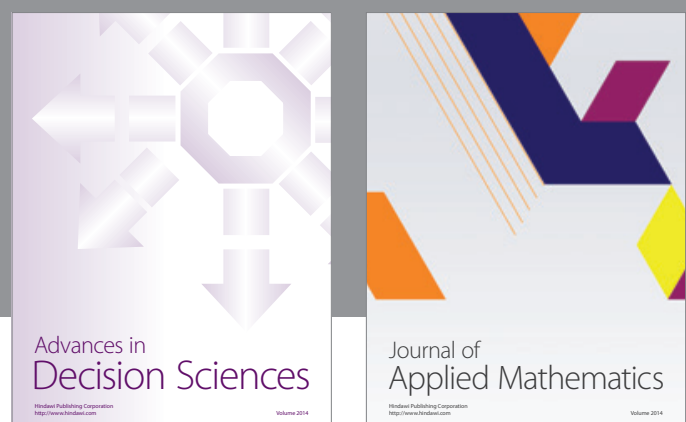

Journal of

Applied Mathematics
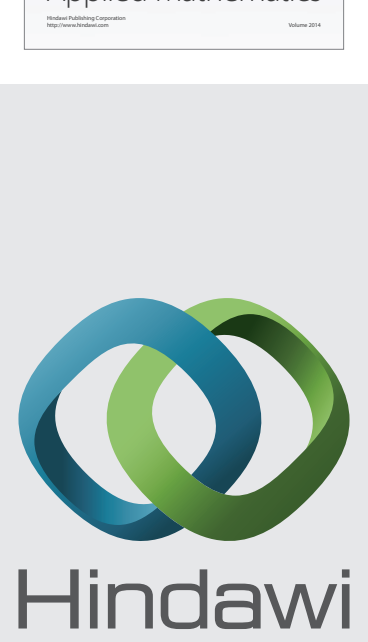

Submit your manuscripts at http://www.hindawi.com
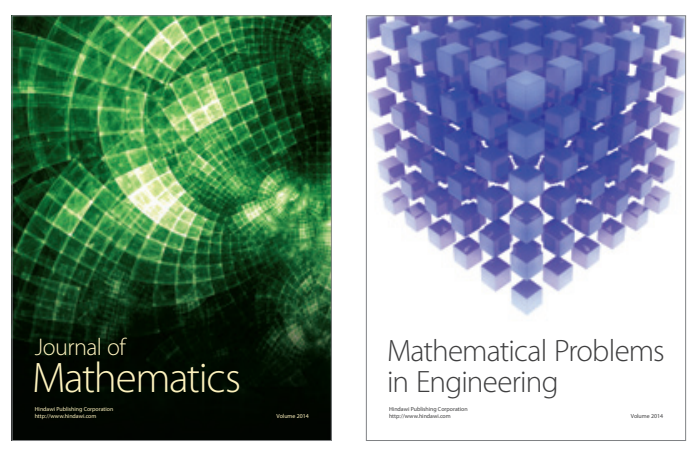

Mathematical Problems in Engineering
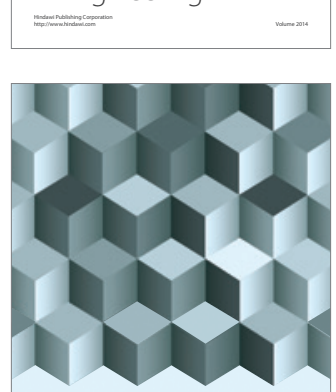

Journal of

Function Spaces
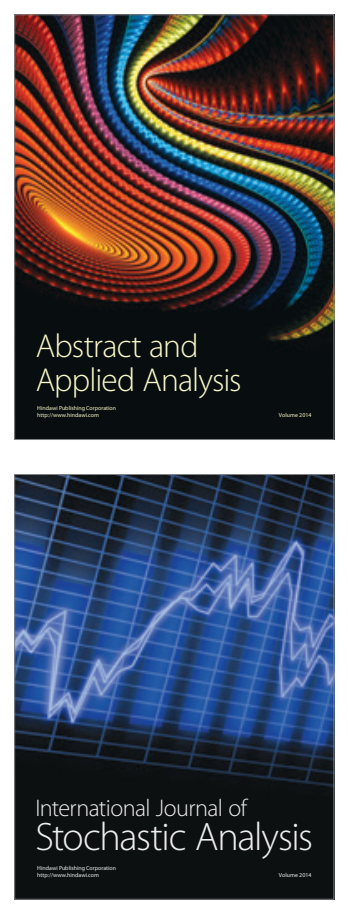

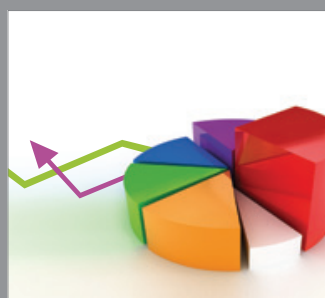

ournal of

Probability and Statistics

Promensencen
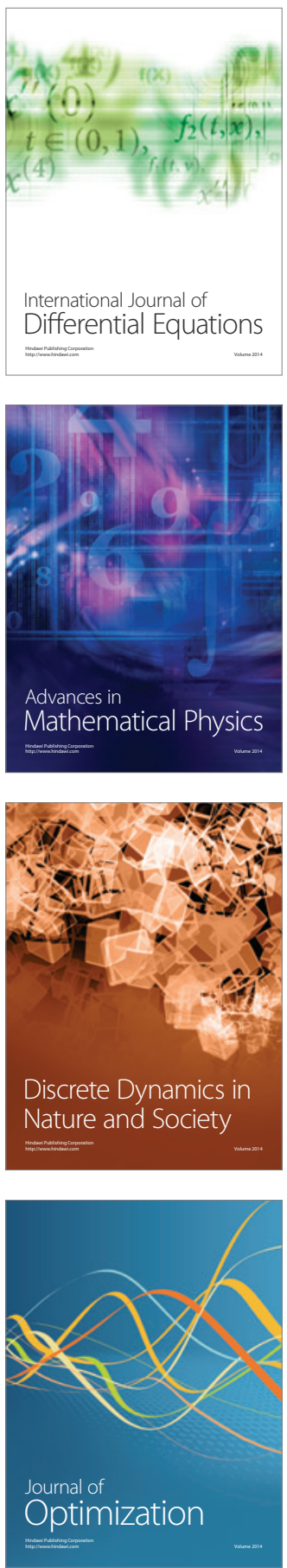